\title{
UNA ESTRATEGIA PARA LOGRAR EL DESARROLLO SOSTENIBLE ENDÓGENO EN AMERICA LATINA Y EL CARIBE
}

\section{A STRATEGY TO ACHIEVE SUSTAINABLE ENDOGENOUS DEVELOPMENT IN LATIN AMERICA AND THE CARIBBEAN}

iD Maribel Enaida Alegre Jara ${ }^{1} \quad$ (iD Lizbeth Dora Briones Pereyra ${ }^{1}$

1Universidad Nacional del Santa, Ancash, Perú

Correspondencia:

Dra. Maribel Enaida Alegre Jara

malegre@uns.edu.pe

\section{RESUMEN}

La mayoría de las personas tienen la necesidad de un cambio en cuanto a lo económico, social y ambiental, por supuesto, con diferentes enfoques, y casi nunca con una visión integral, el objetivo de la presente investigación, fue realizar una revisión sobre, el desarrollo sostenible de los países de América Latina. El estudio se basó, en un enfoque cuanti-cualitativo, tipo básico, aplicándose el método analítico-sintético, en la búsqueda de la información teórica; en la determinación de los fundamentos, en el procesamiento empírico e interpretación de los resultados obtenidos. Así mismo, se analizó por separado, la Sostenibilidad Económica, Ambiental y Social, de manera que, América Latina deba cambiar paradigmas, para lograr el desarrollo sostenible.

Palabras clave: desarrollo sostenible, endógeno, economía circular

\section{ABSTRACT}

Most people have the need for a change in terms of economic, social and environmental, of course, with different approaches, and almost never with a comprehensive vision, the objective of this research was to conduct a review on, the sustainable development of Latin American countries. The study was based on a quantitativequalitative approach, basic type, applying the analytical-synthetic method, in the search for theoretical information; in the determination of the foundations, in the empirical processing and interpretation of the results obtained. Likewise, Economic, Environmental and Social Sustainability was analyzed separately, so that Latin America must change paradigms in order to achieve Sustainable development.

Key words: sustainable development, endogenous, circular economy 


\section{INTRODUCCIÓN}

En la conferencia internacional, celebrada en Tesalónica: Environment and Society: Education and Public Awareness for Sustainability (UNESCO, 1997), el director Mayor Zaragoza, afirmó: "Debemos estar preparados, en todos los países, a replantear la educación con el fin de promover actitudes y conductas propicias a una cultura de la sostenibilidad". Por otra parte, la noción de sostenibilidad en el Informe Brundtland es evidentemente ambivalente (Naredo, 1996), cuya definición es el desarrollo que satisface las necesidades de la generación presente, sin embargo, sin comprometer la capacidad de las generaciones futuras para satisfacer las propias. Los discursos versan sobre un futuro mejor y en ocasiones hablan del Desarrollo Sostenible como al alcance de la mano, pero la realidad es otra, debido a que, los indicadores de la Naciones Unidas CEPAL (2021), muestran que las tendencias, aún antes de la Pandemia, son en América Latina hacia un estancamiento a largo plazo. En este artículo se propone una estrategia basada en el Desarrollo Endógeno Sostenible, con elementos de Economía Circular, para transformar el mito de la sostenibilidad en una meta alcanzable.

\section{MATERIALES Y MÉTODOS}

El estudio se trata de una investigación de enfoque cuanti-cualitativo, tipo básico, Diseño no experimental, correlacional, se aplicaron métodos como el analítico-sintético en la búsqueda de la información teórica; en la determinación de los fundamentos, en el procesamiento empírico e interpretación de los resultados obtenidos y en la elaboración de las conclusiones del trabajo. El proceso de revisión documental tuvo como fuente 42 materiales bibliográficos (informes de Investigación, documentos oficiales de la CEPAL, UNESCO, ONU, artículos científicos, tesis doctorales, libros, boletines, ponencias presentadas en eventos científicos, informaciones estadísticas de América Latina y el Caribe) en los que se exponen, entre otros resultados, estudios diagnósticos, estudios comparados, reflexiones teóricas y propuestas , que aluden a experiencias que engloban referencias a diversos países. El método inductivo-deductivo, permitió establecer generalizaciones sobre sostenibilidad en América Latina, a partir del procesamiento de la información empírica, propiciando el conocimiento sobre la realidad del fenómeno que se investiga. La sistematización permitió la localización, el ordenamiento, la clasificación, el análisis se concretó mediante una estrategia metodológica.

\section{RESULTADOS y DISCUSIÓN Sostenibilidad}

La introducción del término de sustentabilidad se hizo en la declaración de Cocoyot, con motivo de una reunión celebrada por Naciones Unidas en México en 1974, y fue asumida en la publicación de la Estrategia Mundial de la Conservación de la UICN (Unión Internacional de la Conservación de la Naturaleza), en 1980. La concreción, desarrollo y difusión a escala mundial del concepto de desarrollo sostenible lo realizó la Comisión Mundial para el Medio Ambiente y el Desarrollo (CMMAD, 1986).

El desarrollo sostenible es un desarrollo viable en el tiempo, con la condición de que no se base en menguar las posibilidades de las futuras generaciones, todos coincidimos en este reconocimiento, nadie quiere dejar a sus descendientes un lugar inhabitable o una vida miserable, pero el sistema económico se ha concebido siempre desde posiciones extremas como un sistema aislado de su entorno, metafóricamente hablando, como si el sistema circulatorio del cuerpo humano no tuviera vinculación alguna con el digestivo (Daly, 1994).

Hay posiciones antagónicas, cuando se incluye la idea que este accionar económico genera la aparición de problemas ambientales que recaen generalmente sobre personas que no los causan, se habla entonces de la internalización de las externalidades y se requiere de mecanismos que valoren estos daños, se resarza a las víctimas; para ello, debe valorarse monetariamente el medio ambiente natural, de modo que, el mercado no se resienta y que aquel pueda mejorarse 
(Haro Martínez, 2018). Otro punto de vista, tiene Martínez-Alier \& Schlüpmann (1991), de que el medio ambiente es inconmensurable en términos monetarios, no solamente porque se dan en él procesos de un valor vital y ecológico insustituibles, sino también porque las futuras generaciones, que son quienes van a heredar el capital natural, no pueden participar en el juego de la valoración a través de la oferta y de la demanda. También se plantea que dicha internalización debe destinarse a mejorar la calidad de vida de las comunidades involucradas, la protección del medio ambiente y al uso racional de los recursos naturales (Zequeira, 2007).

El concepto de desarrollo sostenible concibe el desarrollo como un proceso armónico, donde la explotación de los recursos, la dirección de las inversiones, la orientación del cambio tecnológico y las transformaciones institucionales deben corresponderse con las necesidades de las generaciones presentes y futuras. Así, se presenta el desarrollo como un proceso que requiere un progreso global, tanto en materia económica y social, como en los órdenes ambiental y humano (Pichs,
2002). Es decir, podemos hablar de:

- Sostenibilidad económica

- Sostenibilidad ambiental

- Sostenibilidad social

La sostenibilidad económica es la capacidad que tiene una organización de administrar sus recursos y generar rentabilidad de manera responsable y en el largo plazo. Surge la oportunidad de gestionar mejor los recursos, y que estos sirvan para, obtener la mayor utilidad posible. Las empresas sostenibles económicamente aplican en todos sus ámbitos de acción productiva este principio.

En buena lid, este es el sueño de empresas y gobiernos, independientemente que en la mayoría de los casos se acompañe de un discurso encendido, sobre "LAS OTRAS" sostenibilidades. Sin embargo, el balance económico de América Latina y el Caribe en 2019, se presentó en un contexto particularmente complejo. La región muestra una desaceleración económica generalizada y sincronizada a nivel de países y de sectores, manteniendo la tendencia de los últimos años de bajo crecimiento, señaló la CEPAL - Chile.

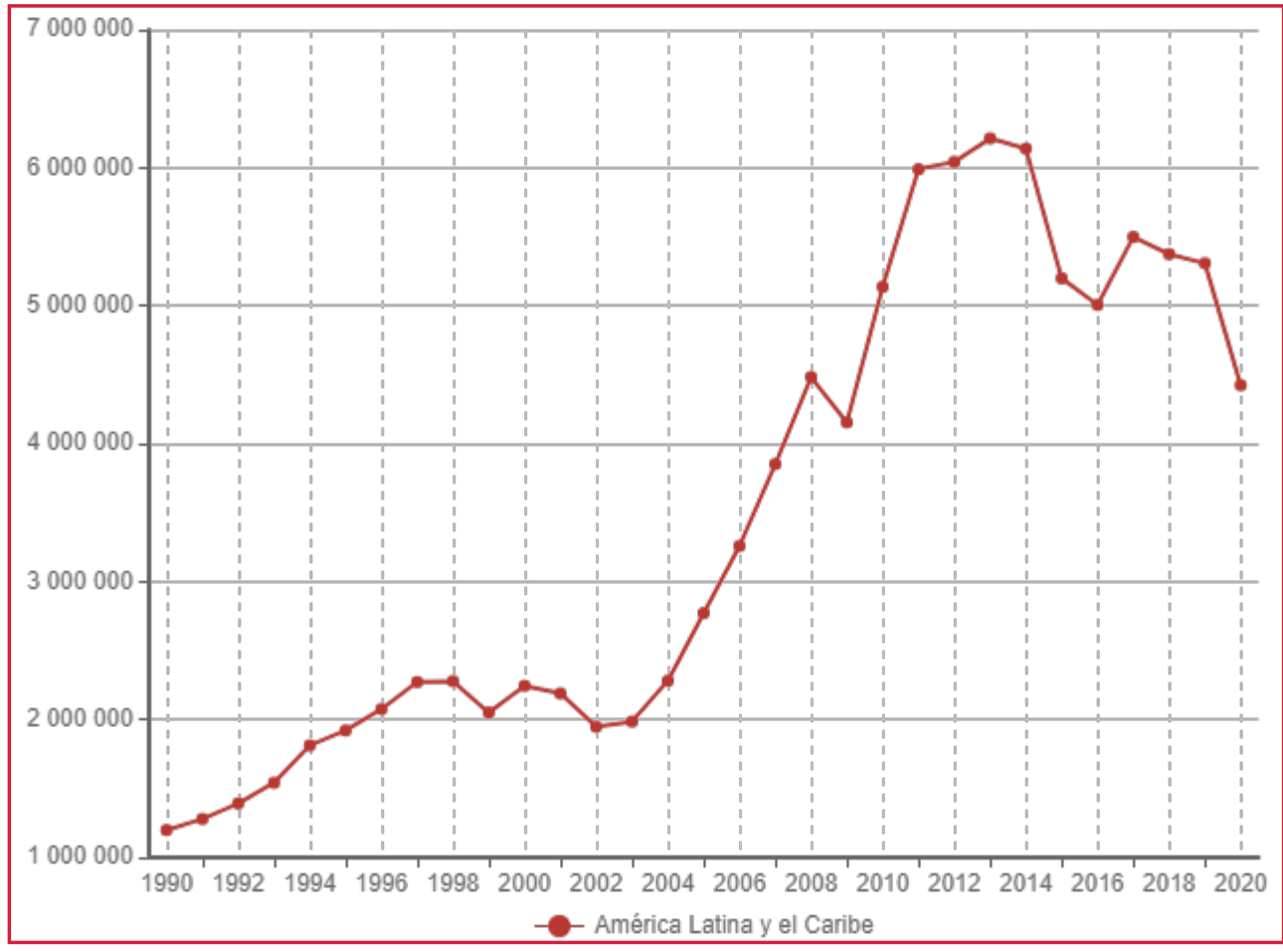

Figura 1. Producto Interno Bruto (PIB) anual por habitante por actividad económica a precios constantes en dólares. Fuente: CEPALSTAT (2021) 
La tendencia en los últimos años del PIB, es decreciente para América Latina, desde un

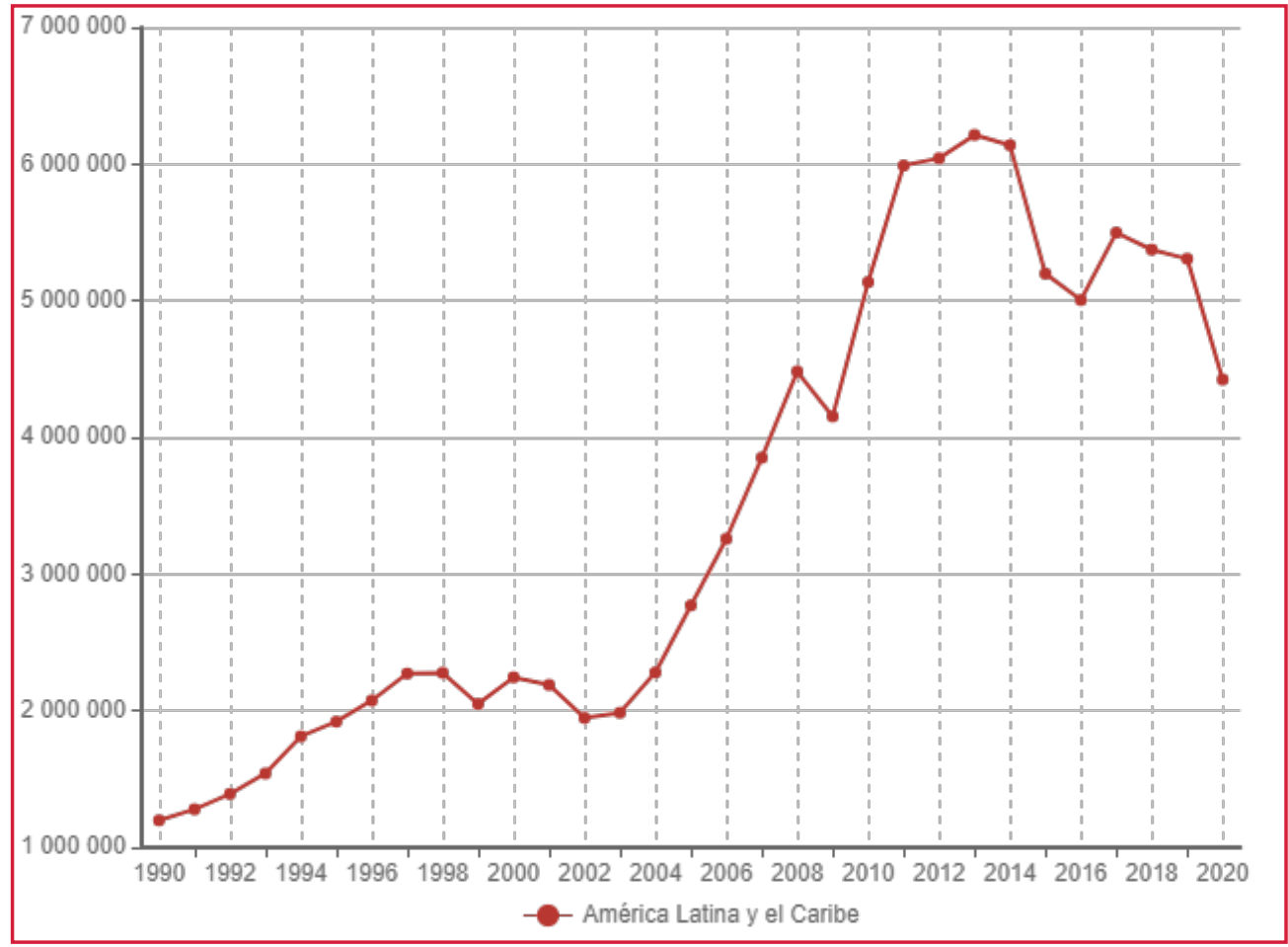

Figura 2. Producto Interno Bruto (PIB) total anual a precios corrientes en dólares (Millones de dólares). Fuente: CEPALSTAT (2021)

En octubre, del 2020, el profesor Pierre Salama de la Universidad de la Sorbona de Paris, planteó que los países de América Latina, tales como, Argentina, Brasil y México, sufren un estancamiento económico de largo plazo, considerando que las causas principales, son: el papel de la desigualdad en materia de ingresos y riqueza, los procesos de desindustrialización, y las causas de la volatilidad económica y sus consecuencias, para la baja tasa media de crecimiento per cápita.

Asimismo, países como Perú, han desarrollado fuertemente su especialización en productos primarios, en ausencia de un tejido industrial importante, lo que podría aumentar el crecimiento del PIB, pero éste sigue siendo vulnerable, porque depende mucho del precio de las materias primas y de las cantidades vendidas, sobre las que el país tiene poco control.

Con este análisis se evidencian dos aspectos, la sostenibilidad económica, la cual no debe verse de forma independiente, sino que, integrarse con lo ambiental y lo social y, la necesidad de cambios que conduzcan al verdadero Desarrollo Sostenible.

\section{La Sostenibilidad Ambiental}

La protección del medio ambiente y de las personas contra los efectos de la degradación ambiental es cada vez más aceptada, como parte integrante de cualquier estrategia de superación de la pobreza y la desigualdad intra-generacional. A nivel nacional, los países de América Latina y el Caribe, enfrentan numerosos y diversos retos de política ambiental, emanados del desafío común de superar la heterogeneidad estructural y la dependencia en ventajas comparativas estáticas de las industrias intensivas en recursos naturales. La elevada desigualdad y segregación económica de la estructura social, resultante de este camino histórico de desarrollo, condujo a la adopción de patrones de consumo caracterizados por la segregación, con mercados privados de gran desarrollo, en el caso de, los bienes de alta calidad y, más notablemente, de servicios 
como el transporte, la salud y la educación. Esta segregación separa a los ricos de los pobres, acentúa las disparidades y obstaculiza la puesta en marcha de soluciones públicas más inclusivas y que contemplen la protección del medio ambiente (CEPAL, 2015).

Las políticas dirigidas específicamente a solucionar problemas ambientales también deben contemplar los desafíos en materia de desigualdad. Se deben poner en marcha mecanismos, para garantizar que las políticas y los instrumentos ambientales adoptados en todos los niveles de gobierno, no generen una carga adicional para las personas pobres y vulnerables, sino que, den prioridad a la protección y el mejoramiento de sus condiciones de vida. (CEPAL 2015).

\section{Biodiversidad}

La biodiversidad cumple un papel determinante en procesos atmosféricos y climáticos a nivel regional y planetario. Cuantas más especies comprende un ecosistema, mayor diferenciación genética posee y más probabilidades tiene de ser estable, resiliente y resistente a cambios extremos. La diversidad biológica es uno de los factores determinantes de los procesos de recuperación, reciclaje y reconversión de nutrientes y desechos.

América Latina y el Caribe poseen una gran diversidad biológica (biodiversidad), con enorme riqueza en variabilidad genética y de comunidades biológicas (ecosistemas). La región alberga casi una cuarta parte de la superficie boscosa del mundo y comprende a seis de los países biológicamente más diversos del mundo (Brasil, Colombia, Ecuador, México, Perú y Venezuela). La región concentra entre el $31 \%$ y el $50 \%$ de las especies de mamíferos, aves, reptiles, anfibios, plantas e insectos (PNUMA, 2010).

Sin embargo, la biodiversidad de la región se ha visto seriamente amenazada, en particular como consecuencia del cambio de uso del suelo, para aumentar las tierras de cultivo y pastizales, lo que ha incrementado la degradación de suelos y la expansión de la desertificación. Una cuarta parte del territorio de la región, se compone de tierras desérticas y áridas. La degradación de estas tierras está repercutiendo en la caída de la productividad biológica de los ecosistemas y en una baja de la productividad económica de la agricultura, la ganadería y la silvicultura. (CEPAL, 2014).

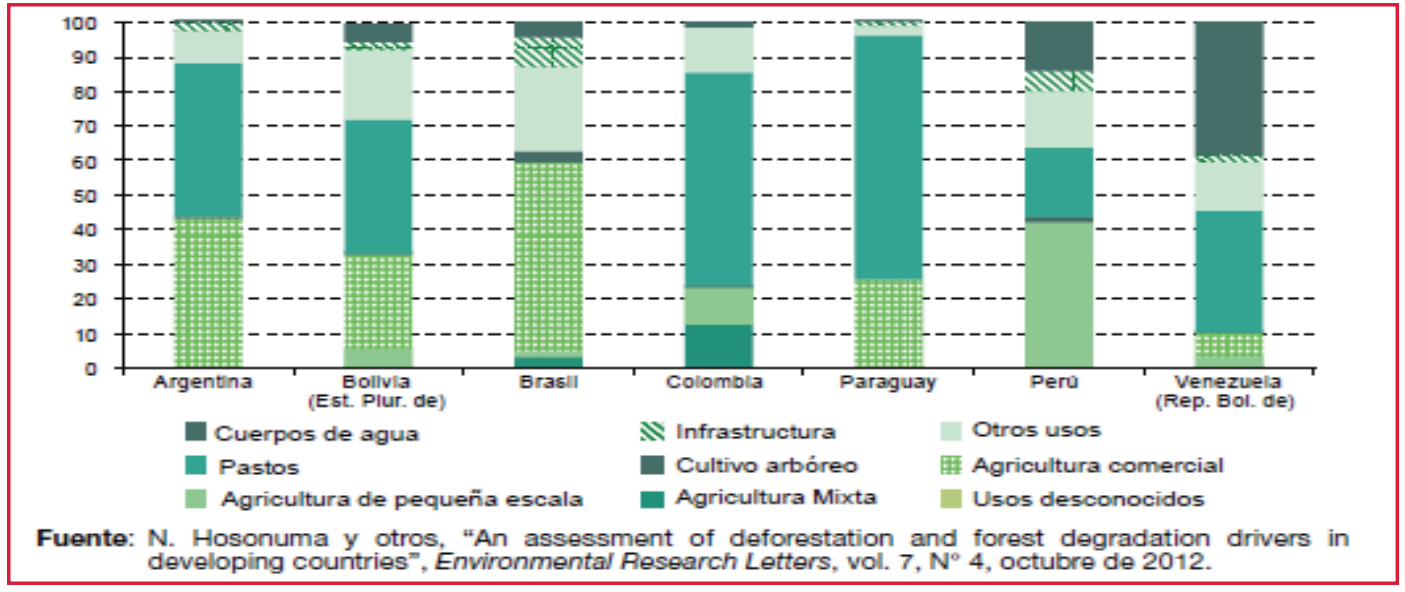

Figura 3. América del Sur (7 países): deforestación atribuida a diversos factores (1990-2005) Fuente: La tragedia ambiental de América Latina y el Caribe.

En materia climática, los cambios están ocurriendo más rápido de lo previsto. Los recientes hallazgos de una comparación cruzada de las predicciones de los principales modelos climáticos muestran que, todos exhiben cambios abruptos en el sistema climático global y que de un total de 37 registran cambios bruscos, cuando el incremento de la temperatura global respecto de la época preindustrial se aproxima al uso irracional de los recursos naturales y sus efectos negativos sobre el medio ambiente y los servicios que 
este presta, desencadenan procesos como la contaminación del aire, el agua y el suelo; el deterioro de cauces y cuencas hidrográficas; así como, la reducción de cuerpos de agua superficiales y acuíferos, y de la biodiversidad asociada; la contaminación y acidificación de los océanos y la pérdida de biodiversidad marina; la deforestación y la pérdida de biodiversidad terrestre; la degradación de las tierras que, con la exacerbación de las sequías, da lugar a la desertificación del calentamiento global y el cambio climático asociado, y, por último, el incremento de la vulnerabilidad de los asentamientos humanos y el riesgo de que ocurran desastres naturales y tecnológicos. Todas estas realidades, que tienen serios impactos productivos, económicos y sociales, están asociadas a las diferentes políticas que cada país adopta para su desarrollo (CEPAL, 2020).

\section{La sostenibilidad Social}

Según el Pacto Mundial de las Naciones Unidas, la sostenibilidad social es una forma proactiva de gestionar e identificar los impactos del negocio en los empleados, trabajadores de la cadena de valor, clientes y comunidades locales. Además, abarca el impacto de las corporaciones en las personas y la sociedad, Incluso, implica un compromiso con la igualdad de género; el empoderamiento de las mujeres, el fomento de la participación de la población de las áreas menos favorecidas y el respeto a los derechos de los niños. Más allá del lugar de trabajo, incluye la lucha contra la pobreza y la desigualdad, la promoción de la educación, la salud, la paz y la estabilidad social. 


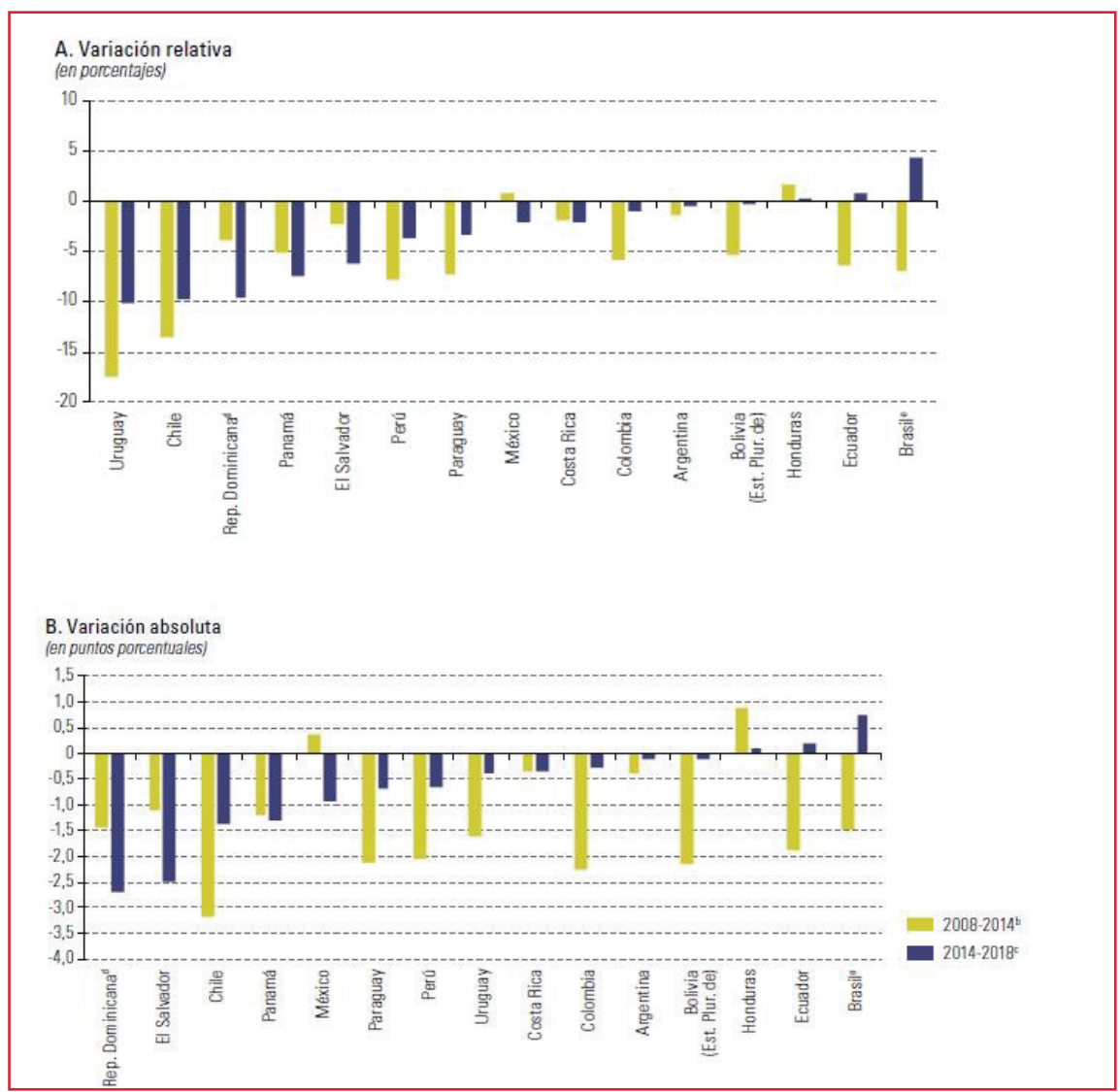

Fuente: Comisión Económica para América Latina y el Caribe (CEPAL), sobre la base de la encuesta de hogares (BADEHOG).

a La variación relativa responde a la variación porcentual de la tasa de pobreza entre los años inicial y final. La variación absoluta es la diferencia en puntos porcentuales entre ambos años.

${ }^{b}$ Los datos corresponden a las variaciones entre (2008 y 2014), excepto en chile (2009 y 2013), Costa Rica (2010 y 2014), El Salvador y Honduras (2009 y 2014). ' Los datos corresponden a las variaciones entre (2014 y 2018), excepto en Chile (2013 y 2017).

d Las cifras no son estrictamente comparables en el período analizado.

e Las cifras de 2008 y 2014 están ajustadas según diferencia entre la encuesta nacional de hogares (PNAD) y la encuesta nacional permanente de hogares (PNAD Continua) de 2014.

Figura 4. América Latina (15 países): variación anualizada de la tasa de pobreza, valores relativos y absolutos 2008 al $2014^{\mathrm{b}}$ y 2014 a $2018^{c}$.

Fuente: Panorama Social de América Latina 2019.

La tendencia a un mejor desempeño en el período 2008-2014b en comparación con el período 2014-2018c, fue más marcada en el caso de la pobreza extrema, puesto que diez países tuvieron un mejor desempeño en el primero. En Brasil y Argentina, la situación empeoró entre 2014 y $2018^{\circ}$ en comparación con el período 2008-2014 ${ }^{\mathrm{b}}$. En Brasil, la pobreza total aumentó a un 4,3\% anual entre 2014 y 2018 ${ }^{\circ}$, lo que contrasta mucho con la tendencia a la baja apreciada entre 2008 y 2014 b (un 6,9 \% anual).
Esto es solo una muestra de que la tendencia no es precisamente a la sostenibilidad social y, no se incluyó en el análisis, la situación generada por la pandemia de COVID-19, que, por supuesto agudizo la pobreza, y generó polarización de la riqueza. 


\section{DISCUSIÓN}

En el resultado de esta investigación se considera inobjetable, que debe trabajarse por un Desarrollo Sostenible, que integre de forma armónica, económico, medioambiental y social.

La comprensión de este enfoque, conduce a la interpretación de la compleja realidad y el reconocimiento del desarrollo como un proceso permeado de relaciones de interdependencia y complementariedad; que tiene relación con los tres objetivos generales y los principios para definir el desarrollo sostenible (Kopfmüller et al., 2001).

\section{Tabla 1}

\section{La estrategia debe establecer relaciones de equilibrio entre los agentes que interactúan económica y socialmente en el mismo entorno}

\begin{tabular}{|c|c|c|c|}
\hline Objetivos & Asegurar la existencia humana & $\begin{array}{l}\text { Mantener la potencia } \\
\text { Productiva de la sociedad }\end{array}$ & $\begin{array}{l}\text { Mantener las opciones de } \\
\text { desarrollo y actuación de la } \\
\text { sociedad }\end{array}$ \\
\hline \multirow{5}{*}{ Principios } & Protección de la salud humana & $\begin{array}{l}\text { El uso sostenible de recursos } \\
\text { naturales renovables }\end{array}$ & $\begin{array}{l}\text { La igualdad de oportunidades en } \\
\text { educación, empleo e información }\end{array}$ \\
\hline & $\begin{array}{l}\text { Satisfacción de las } \\
\text { necesidades básicas }\end{array}$ & $\begin{array}{l}\text { El uso sostenible de recursos } \\
\text { naturales no renovables }\end{array}$ & $\begin{array}{l}\text { La participación en los procesos } \\
\text { sociales de decisión }\end{array}$ \\
\hline & $\begin{array}{l}\text { La Posibilidad de las personas } \\
\text { de asegurar autónomamente su } \\
\text { existencia }\end{array}$ & $\begin{array}{l}\text { El uso sostenible del medio } \\
\text { ambiente como receptor de } \\
\text { emisiones }\end{array}$ & $\begin{array}{l}\text { La protección de la herencia } \\
\text { cultural y de la diversidad cultural }\end{array}$ \\
\hline & $\begin{array}{l}\text { La distribución justa del } \\
\text { acceso a los recursos } \\
\text { naturales y de uso }\end{array}$ & $\begin{array}{l}\text { Evitar } \quad \text { riesgos } \\
\text { tecnológicos inaceptable }\end{array}$ & $\begin{array}{l}\text { La protección de la función cultural } \\
\text { de la naturaleza }\end{array}$ \\
\hline & $\begin{array}{l}\text { El equilibrio de las diferencias } \\
\text { extremas entre ingresos y } \\
\text { propiedad de bienes }\end{array}$ & $\begin{array}{l}\text { El desarrollo sostenible del } \\
\text { capital material, humano y } \\
\text { de conocimiento }\end{array}$ & $\begin{array}{l}\text { Asegurar los } \\
\text { capacidades } \\
\text { ".sociales" }\end{array}$ \\
\hline
\end{tabular}

Savitz y Weber (2006), abarcan tres medidas de sostenibilidad, son ellas: social - solo habrá sostenibilidad social al desarrollarse prácticas laborales, alcanzar la justicia social generando renta y oportunidades, por medio de los derechos humanos; económico - para ser económicamente sostenible debe generar retorno de la inversión (ROI), pagar impuestos, evitar problemas en los flujos monetarios y generar puestos de trabajo; ambiental - la sostenibilidad ambiental busca mantener la calidad del aire, del agua, y de la energía y no comprometer las bases de los recursos, renovables o no renovables.

Sin embargo, una enseñanza de la Pandemia, con respecto al Desarrollo Sostenible, es que este no solo tiene que ser armónico e integral, a su vez debe tener una base Endógena, es decir; El desarrollo endógeno sostenible, parte de un conocimiento de las potencialidades eco-sistémicas, el enfoque eco-sistémico $(E E)$, es una estrategia para el ordenamiento integrado de la tierra el agua y los recursos vivos que promueve la conservación y el uso de la biodiversidad (Toure Sene, 2016); teniendo en cuenta los principios enunciados, quien lo puede conducir al éxito o el fracaso, son las personas y las relaciones que puedan crear.

Para alcanzar un desarrollo local en contextos de continuos cambios, se requiere fomentar la innovación, la capacidad emprendedora, la calidad del capital humano y la flexibilidad de los sistemas productivos (Méndez \& Lloret, 2004). 
La elaboración de cualquier estrategia de desarrollo, requiere conocer las características de la población, sus necesidades, preferencias, potencialidades, los recursos materiales disponibles y la situación de sus recursos naturales; ello implicará el uso del espacio físico, la gestión económica y la valorización de la iniciativa comunitaria como elementos sustantivos del desarrollo a nivel local (Miranda et al., 2007).

Uno de los requisitos para lograr el desarrollo, es el encadenamiento productivo, que impulsa a cada eslabón de la cadena productiva a trabajar coordinadamente en la consecución de los planes comunes, concretos y medibles, que se reflejarán directa y proporcionalmente en los resultados individuales (Ortecho et al., 2020).

Para que el desarrollo sostenible posea la máxima dimensión, se ha retomado la idea de los años 90 enunciada por Pearce y Turner (1990), la "Economía Circular", proponiendo un flujo económico cerrado que explicaba que funcionaría como un metabolismo en cada empresa y luego la formación de simbiosis industrial entre organizaciones distintas. Así, el refuerzo de la idea de cerrar los flujos de energía y materiales, es congruente con la evolución reciente del concepto de sostenibilidad. Mebratu (1998), indica que las tres dimensiones de la sostenibilidad (económica, social y ambiental), no solo deben integrarse, sino que, deben estar configuradas como una sola unidad donde todo lo económico afecta lo social, y todo lo social a lo ambiental, por tanto, todo fenómeno ambiental afecta las dimensiones social y económica de la sostenibilidad.

Un elemento que ha sido muy utilizado como discurso político, pero a integrar de forma definitiva en el desarrollo endógeno sostenible, es la Soberanía Alimentaria, Se entiende por Soberanía Alimentaria: "el derecho de cada pueblo, comunidad y país a definir sus propias políticas agrícolas, pastoriles, laborales, de pesca, alimentarias y agrarias que sean ecológicas, sociales, económicas y culturalmente apropiadas a sus circunstancias exclusivas. Esto incluye el derecho real a la alimentación y a la producción de alimentos, lo que significa que todos los pueblos tienen el derecho de tener alimentos y recursos, para la producción de alimentos seguros, nutritivos y culturalmente apropiados; así como, la capacidad de mantenerse a sí mismos y a sus sociedades" (ONG/OSC, 2002). Consideramos que este es uno de los primeros pasos (muy difícil), para que la Sostenibilidad deje de ser un Mito, y se transforme en una Meta.

\section{CONCLUSIONES}

- El desarrollo sostenible es un desarrollo viable en el tiempo, con la condición de que no se base en menguar las posibilidades de las futuras generaciones. Se considera como un proceso que requiere un progreso global, tanto en materia económica y social como en los órdenes ambiental y humano.

- El análisis de la Sostenibilidad de forma independiente indica que América Latina, requiere un cambio radical, pues no se están logrando resultados que este conduciendo a un desarrollo sostenible.

- La estrategia que se propone debe basarse en el Desarrollo Endógeno Sostenible tomando los paradigmas de la Economía Circular y tomando como elemento central la Soberanía Alimentaria.

\section{REFERENCIAS BIBLIOGRÁFICAS}

CEPAL (2015). El desafío de la sostenibilidad ambiental en América Latina y el Caribe. Disponible en: https://repositorio.cepal. org/bitstream/handle/11362/37791/ LCM23_es.pdf

CEPAL (2020). La tragedia ambiental de América Latina y el Caribe. Disponible en: https://repositorio.cepal.org/bitstream/ handle/11362/46101/1/S2000555_ es.pdf

CEPAL (2019). Panorama Social de América Latina. Disponible en: https:// repositorio.cepal.org/bitstream/ handle/11362/44969/5/S1901133_ es.pdf 
CEPALSTAT (2021). Base de datos y Publicaciones Estadísticas. Disponible en: https://statistics.cepal.org/ portal/cepalstat/dashboard. html? theme=2\&lang $=$ es

CMMAD (1986). Desarrollo y cooperación económica internacional: Medio Ambiente. Disponible en: http:// www. ecominga.uqam.ca/PDF/ BIBLIOGRAPHIE/GUIDE_LECTURE_1/ CMMAD-Informe-Comision-Brundtlandsobre-Medio-Ambiente-Desarrollo.pdf

Daly, H.E. (1994). Los peligros del libre comercio. Investigación y Ciencia, 12-41. Disponible en: https://www. investigacionyciencia.es/revistas/ investigacion-y-ciencia/conmutadorde-silicio-79/Los-peligros-del-librecomercio-5662

Haro-Martínez, A. \& Taddei-Bringas, I. (2014). Sustentabilidad y economía: la controversia de la valoración ambiental. Economía, Sociedad y Territorio, XIV (46),743-767. Disponible en: https://www. redalyc.org/articulo.oa?id=11131650006

Kopfmüller, J., Brandl, V., Jörissen, J., Paetau, M., Banse, G., Coenen, R., \& Grunwald, A. (2001). Nachhaltige Entwicklung integrativ betrachtet - Konstitutive Elemente, Regeln, Indikatoren (desarrollo sostenible visto integralmente elementos constitutivos, normas, indicadores). Edition Sigma. Berlin. 432 p.

Martínez-Alier, J. \& Schlüpmann, K. (1991). La ecología y la economía. México: Fondo de Cultura Económica.

Mebratu (1998). Sustainability and sustainable development: Historical and conceptual review. Environmental Impact Assessment Review, 18 (6), 493 - 520. DOl: $\quad$ https://doi.org/10.1016/S01959255(98)00019-5

Méndez, E. \& Lloret, M. C. (2004). ¿Cómo medir el desarrollo local según la experiencia cubana? Observatorio de la Economía Latinoamericana, (29), 1-19. Dispoible en https://ideas.repec.org/a/ erv/observ/y200429mell1.html

Miranda, T., Suset, A., Cruz, A., Machado, H. \& Campos, M. (2007). El Desarrollo sostenible. Perspectivas y enfoques en una nueva época. Pastos y Forrajes, 30(2), 191-204. DOI: 10.1016/j. jbusvent.2008.01.001

Naciones Unidas - CEPAL (2021). Construir un futuro mejor: Acciones para fortalecer la Agenda 2030 para el Desarrollo Sostenible. Disponible en: https://repositorio.cepal.org/bitstream/ handle/11362/46682/6/S2100125_ es.pdf

Naredo, J. (2001). Economía y sostenibilidad. La economía ecológica en perspectiva. POLIS, Revista Latinoamericana, 1(2). 1-28. Disponible en: https://www.redalyc. org/articulo.oa? $\mathrm{id}=30500213$

NGO/CSO (2002). Forum for Food Sovereignty. Roma. Disponible en: http://www.ukabc. org/wfs5+.htm

Ortecho, R., Medina, D., Sánchez, A., Manayay, D., P., G., \& Taramona, L. (2020). Modelo de actuación para el desarrollo endógeno sostenible en Tayacaja, Perú. Retos de la Dirección, 14(2), 274-300. Disponible en: http://scielo.sld.cu/scielo. php?script=sci_arttext $\&$ pid=S230691552020000200274\&lng=es\&tlng=pt

Pearce, D.W, y Turner, R.K. (1990). Economics of the Natural Resources and the Environment. Harvester Wheatsheaf, London, UK.

Pichs, R. (2002). Los retos del desarrollo sostenible en América Latina. Disponible en: http://www.redem.buap.mx/ramon. htm.

PNUMA (2010). Perspectivas del Medio Ambiente Mundial: Opciones de 
política para América Latina y el Caribe. Disponible en: http://www.pnuma.org/ deat1/Policy\%20Briefs/Espanol/Brief_ governance_espanol(webpags).pdf

Salama, P. (2020). ¿Por qué los países latinoamericanos sufren un estancamiento económico de largo plazo? Un estudio a partir de los casos de Argentina, Brasil y México. El trimestre económico, 87(348), 10831132. DOI: https://doi.org/10.20430/ete. v87i348.1167

Savitz, A. \& Weber, K. (2006): The triple bottom line: how today's best run companies are achieving economic, social and environmental success - and how you can too. San Francisco: Jossey Bass.

Toure Sene, E.H.O. (2016). Enfoque eco sistémico del territorio para una gestión integrada del delta del Río Senegal [Tesis de doctorado]. Universidad Autónoma de Barcelona (UAB). Disponible en: https://www.tdx.cat/bitstream/ handle/10803/400709/ehots1de1. pdf? sequence $=1 \&$ is Allowed $=y$

UNESCO (1997). Environment and Society: Education and Public Awareness for Sustainability. Salónica, Grecia. Disponible en: https://www.iau-hesd. net/sites/default/files/documents/ thessaloniki.pdf

Zequeira, M. (2007). Instrumento económico y metodológico para le gestión ambiental en humedales naturales cubanos con interés internacional [Tesis de doctorado]. Universidad de Camagüey, Cuba. 